\title{
The Effect of Fibroblast Growth Factors in Grafted Fascia into the Vocal Fold of Rabbits
}

\author{
Eduardo G. B. Carvalho ${ }^{1}$ Almiro J. Machado-Júnior ${ }^{1}$ Henrique F. Pauna ${ }^{1}$ Ester M.D. Nicola ${ }^{1}$ \\ Albina M.A.M. Altemani ${ }^{2}$ Agricio N. Crespo ${ }^{1}$ \\ ${ }^{1}$ Department of Otorhinolaryngology, Head and Neck Surgery, \\ Universidade Estadual de Campinas, Campinas, SP, Brazil \\ 2 Department of Pathology, Universidade Estadual de Campinas, \\ Campinas, SP, Brazil

\begin{abstract}
Address for correspondence Henrique Furlan Pauna, Departamento de Otorrinolaringologia e Cirurgia de Cabeça e Pescoço, Universidade Estadual de Campinas, Rua Tessália Vieira de Camargo, 126, Campinas, SP, 13083-887, Brazil (e-mail: h_pauna@hotmail.com).
\end{abstract}

Int Arch Otorhinolaryngol 2019;23:60-64.

\begin{abstract}
Keywords

- vocal folds

- rabbits

- FGFs

- fibroblasts

- grafting

- fascia

Introduction The human larynx is a very important organ for communication. Many conditions lead to scarring of the vocal folds, decreasing voice quality.

Objective We aimed to determine whether fibroblast growth factors (FGFs) may influence tissue integration of grafted fascia into the vocal folds of an animal model. Methods This is an experimental animal study with 12 adult rabbits that were submitted to a grafting fragment obtained from superficial cervical fascia into the vocal fold lamina propria, bilaterally. The right vocal fold was injected with FGFs. The animals were sacrificed after 1 month or 12 months, depending on the group they were assigned to, and a histological analysis of their vocal folds was performed. We analyzed the histological changes (such as the presence of fibrosis and neovascularization) induced by the acute or chronic inflammatory reactions.

Results The FGFs induced acute inflammatory changes in all animals after 1 month of the initial experiment. The presence of FGFs triggered more fibrosis than the expected due to the surgical procedure itself when compared with the control side of all animals after 12 months of the initial experiment.

Conclusions Fibroblast growth factors alone do not represent a good therapeutic option in phonosurgery, since we observed higher levels of fibrosis in the vocal fold lamina propria. Further studies combining more substances may be necessary to elucidate the best option to be used in this kind of surgery.
\end{abstract}

\section{Introduction}

The human larynx enables interpersonal interaction. No other organ of the human body presents similar structural arrangement like the vocal folds, especially the flexibility and vibratory features, which are related to sound production. ${ }^{1}$

Several conditions promote structural changes of the vocal fold lamina propria, with the formation of scar tissue (increased deposition of type I collagen and fibronectin, decreased elastin, decorin, and hyaluronic acid), decreasing the vibration, leading to a poorer voice quality. ${ }^{2-4}$ Since the 1990s, many studies focused on reducing postoperative fibrosis and scarring, and to improving the flexibility of the vocal folds. ${ }^{5-11}$ However, many of these techniques are not yet available for clinical usage.

Fibroblast growth factors (FGFs) are potent regulators of cell proliferation, differentiation and function and are critically important to normal development, tissue maintenance, wound repair and angiogenesis. Fibroblast growth factors are also linked with several pathological conditions. ${ }^{12}$ Therefore, we aim to determine whether FGFs may influence tissue integration of grafted fascia into rabbits' vocal folds. received

September 1, 2017

accepted

May 8, 2018

published online

July 5, 2018
DOI https://doi.org/

10.1055/s-0038-1661399. ISSN 1809-9777.
Copyright $\odot 2019$ by Thieme Revinter

Publicações Ltda, Rio de Janeiro, Brazil
License terms

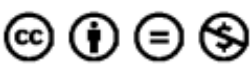




\section{Method}

\section{Ethics}

The Institutional Review Board approved this study (CEP 1033-1).

\section{Specimens}

Twenty New Zealand rabbits of both genres were used in this experiment, weighing between two and three kilograms. The number of male and female animals was the same. All the animals were kept with water and food "ad libitum," from the moment of the procedure to the moment of their sacrifice.

The rabbits were divided into two groups. Group A, with animals maintained under observation for 12 months after the surgical procedure, and group $B$, which was observed for 1 month. The animals were randomly assigned to each group.

Animals with congenital malformations of the larynx and female rabbits that got pregnant during the experiment were excluded from this study.

\section{Surgical Procedure}

The animals underwent intramuscular anesthesia with ketamine (25 mg/kg/dose) and xylazine ( $3 \mathrm{mg} / \mathrm{kg} /$ dose) and kept with spontaneous breathing.

A 4-cm cervical longitudinal incision was performed at the level of the thyroid cartilage. The subcutaneous tissue was exposed by using scissors, and a fragment of superficial fascia of the neck, of $\sim 10 \times 10 \mathrm{~mm}$, was excised. The fascia was extended on a metal surface and left to dehydrate spontaneously.

A 2-mm longitudinal incision was made along the vocal process of the vestibular face of the right and left vocal folds, close to the glottic aperture. A 45 degree-angled dissector was introduced into the incision to create a submucosal pouch, parallel to the medial face of the vocal fold, $\sim 2 \mathrm{~mm}$ long anteroposteriorly, in both right and left vocal folds.

After dehydration, a fragment of $1 \times 1 \mathrm{~mm}$ of the fascia was inserted into the submucosal pouch. The right vocal fold was injected with $5 \mu \mathrm{L}$ of FGFs solution (concentration of 1 $\mathrm{ng} / \mu \mathrm{L}$, diluted in sodium chloride) immediately after the fascia was implanted. The left vocal fold received no injection of FGFs and remained as a control for histological changes due to grafting the fascia.

\section{Histological Analysis}

After the animals were sacrificed, their larynxes were obtained and maintained in a $10 \%$ formalin solution for 24 hours. The vocal folds were then resected with the thyroid cartilage, the segments were histologically processed ( $3 \mu \mathrm{m}$ of thickness for each section) and embedded in paraffin. Every section contained both vocal folds for comparison. The slides were stained with hematoxylin and eosin ( $\mathrm{H}$ and $\mathrm{E})$, picrosirius red, and Masson trichrome and then examined under light microscopy (in different magnifications) to assess the following parameters: a) number and type of inflammatory cells (neutrophils, lymphocytes, and macrophages); b) presence or absence of fibrosis; c) neovascularization; d) the density of collagen within the grafted area; and e) presence or absence of foreign body reaction.
The inflammatory reaction was semiquantitatively evaluated by comparing the small portion of the surrounding of the implanted fascia with the lamina propria beyond this area. It was then classified as:

0-absence of mononuclear cells;

I -mild (1 to 10 mature mononuclear cells were observed);

II -moderate (11 to 20 mature mononuclear cells were observed); and

III -severe (more than 20 mature mononuclear cells).

Additionally, the inflammatory reaction was classified as acute when there was presence of polymorphonuclear cells, and as chronic when there was presence of a foreign body reaction with active macrophage predominance.

Fibrosis and the density of collagen within it were analyzed using a Sony DXC-101/Trinitron CCD Camera System (Sony Electronics Inc., San Diego, CA, USA), scanned trough an OCULUS TCX System (Teledyne DALSA, Waterloo, ON, Canada), and analyzed with the image editing software Image Plus 4.5 for Windows (Astra Image, Kawanishi City, Japan). Fibrosis was also semiquantitatively assessed and graded as:

0-absence of collagen fibers;

I - mild (few foci of collagen fibers);

II - moderate (continued collagen fibers around the grafted area); or

III - severe (bigger area of continued collagen fibers around the grafted area, consisting a fibrous capsule).

Neovascularization was defined by the presence of thin wall blood vessels within the grafted area.

\section{Statistical Analysis}

We calculated and compared the data obtained from the analysis of the inflammatory reaction, presence of neovascularization, and fibrosis using the Fisher exact test. Statistical analysis obtained from the density of collagen fibers was performed by non-parametric $t$-test, Wilcoxon test, and Kruskal-Wallis algorithm, comparing both the left and right sides, and in both groups (A and B). Findings were considered statistically significant when $p$ values were $<0.05$.

\section{Results}

Our final groups comprised of 6 rabbits in group $A$ (12-months survival) and 6 rabbits in group $B$ (1-month survival). All data obtained from the histological analysis are shown in - Table 1.

Histological analysis of cellular predominance in both vocal folds (injected with FGFs or control) revealed predominant lymphohistiocytic infiltration with no differences between sides or groups. Additionally, the lymphohistiocytic pattern was statistically significant when compared with the other types of cells within the inflammation $(p=0.008)$.

There was a statistical difference between both groups when we compared the presence of inflammatory reaction and the side of the vocal fold. The intensity of inflammatory reaction in the right vocal fold (with FGFs injection) showed frequent association with a "moderate" or "severe" reaction in group B; however, in group $A$, it was related to an "absent" or "mild" reaction ( $p=0.011)$ ( - Figs. 1 and 2 ). The intensity 
Table 1 Histological findings in both groups

\begin{tabular}{|c|c|c|c|c|c|c|c|c|c|c|}
\hline \multirow[t]{2}{*}{$\begin{array}{l}\text { Case No./ } \\
\text { Group }\end{array}$} & \multicolumn{2}{|c|}{$\begin{array}{l}\text { Intensity of } \\
\text { inflammatory } \\
\text { reaction }\end{array}$} & \multicolumn{2}{|c|}{ Cell type } & \multicolumn{2}{|c|}{ Fibrosis } & \multicolumn{2}{|c|}{$\begin{array}{l}\text { Chronic } \\
\text { reaction }\end{array}$} & \multicolumn{2}{|c|}{$\begin{array}{l}\text { Neovas- } \\
\text { cularization }\end{array}$} \\
\hline & RVF & LVF & RVF & LVF & RVF & LVF & RVF & LVF & RVF & LVF \\
\hline $1 / \mathrm{A}$ & 1 & 1 & LH & 0 & II & 0 & 0 & 0 & 1 & 0 \\
\hline $2 / \mathrm{A}$ & 1 & 1 & LH & 0 & 1 & 0 & 0 & 0 & 1 & 0 \\
\hline $3 / \mathrm{A}$ & II & I & $\mathrm{LH}$ & 0 & 1 & 0 & 0 & 0 & 1 & 0 \\
\hline $4 / A$ & 1 & I & $\mathrm{LH}$ & 0 & 1 & 0 & 0 & 0 & 1 & 0 \\
\hline $5 / A$ & 1 & I & $\mathrm{L}$ & 0 & 1 & 0 & 0 & 0 & 1 & 0 \\
\hline $6 / A$ & 1 & 1 & $\mathrm{~L}$ & 0 & 1 & 0 & 0 & 0 & 1 & 0 \\
\hline $1 / \mathrm{B}$ & II & 0 & $\mathrm{H}$ & $\mathrm{LH}$ & 0 & 0 & 0 & 0 & 0 & 0 \\
\hline $2 / B$ & II & I & $\mathrm{LH}$ & L & 0 & 0 & 0 & 0 & 0 & 0 \\
\hline $3 / B$ & III & 1 & $L$ & $\mathrm{LH}$ & 0 & 0 & 0 & 0 & 0 & 0 \\
\hline $4 / B$ & II & II & $\mathrm{LH}$ & $\mathrm{LH}$ & 0 & 0 & 0 & 0 & 0 & 0 \\
\hline $5 / B$ & III & 1 & $\mathrm{LH}$ & $\mathrm{LH}$ & 0 & 0 & 0 & 0 & 0 & 0 \\
\hline $6 / B$ & III & 1 & LH & $\mathrm{LH}$ & 0 & 0 & 0 & 0 & 0 & 0 \\
\hline
\end{tabular}

Abbreviations: H, histiocytic pattern; L, lymphocytic pattern; LH, lymphohistiocytic pattern; LVF, left vocal fold; RVF, right vocal fold.

Note: 0 , absence; I, mild; II, moderate; III, severe.

of inflammatory reaction in the left vocal fold (considered as a control group) showed no differences between both groups $(p=0.573)$.

The intensity of fibrosis was statistically significant when we compared the right vocal fold in both groups $(p=0.014)$. It was frequently found in group $A$, and considered as "mild" in most of the samples. The left vocal fold in both groups showed no fibrosis between both groups (-Figs. 3 and 4).

Neovascularization was only observed in the right vocal folds (injected with FGFs) of animals from group A. However, it was not statistically significant between the groups.

None of the vocal folds among both groups showed signs of chronic inflammatory reaction, presence of giant cells, or foreign body reaction.

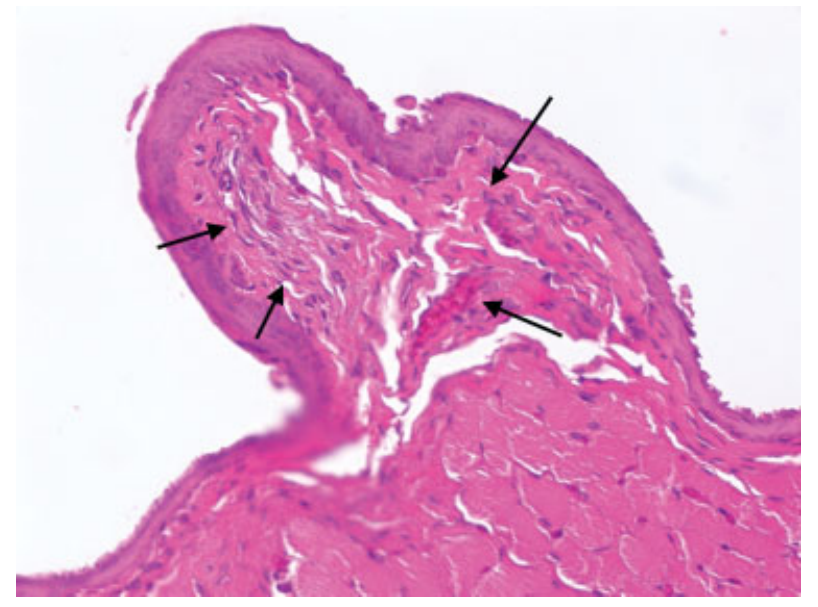

Fig. 1 Right vocal fold of a rabbit from group $A$ with grafted fascia within the lamina propria and injected fibroblasts growth factor. Mild inflammatory reaction (arrow) (hematoxylin and eosin x20).

\section{Discussion}

Many techniques aim to restore the functional mechanism and provide a better oscillation of the vocal fold's mucosa, such as: vocal therapy, injections of collagen or hyaluronic acid, axial incisions of the mucosa, excision of the scarring tissue; additionally, another option consists in the insertion of foreign tissue, such as fat or muscular fascia between the epithelium and the vocal ligament. ${ }^{13-17}$ Fascia graft has been used for decades in otologic and plastic surgeries without complications and with reproducible results. Fascia presents a similar consistency to that of collagen and low rate of metabolic activity. Rihkanen ${ }^{18}$ proposed the use of minced fascia in the surgery of volumetric augmentation of vocal

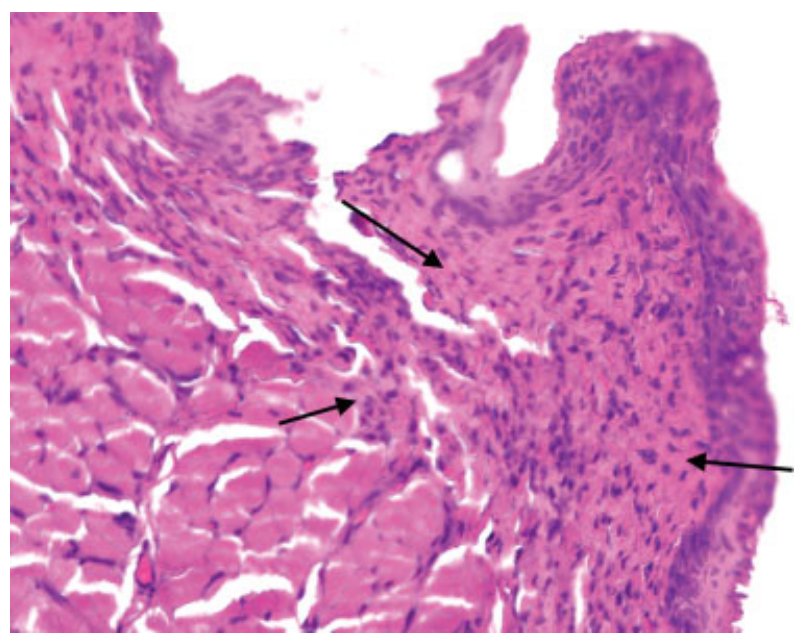

Fig. 2 Right vocal fold of a rabbit from group $B$ with grafted fascia within the lamina propria and injected fibroblasts growth factor. Moderate inflammatory reaction (arrow) (hematoxylin and eosin x20). 


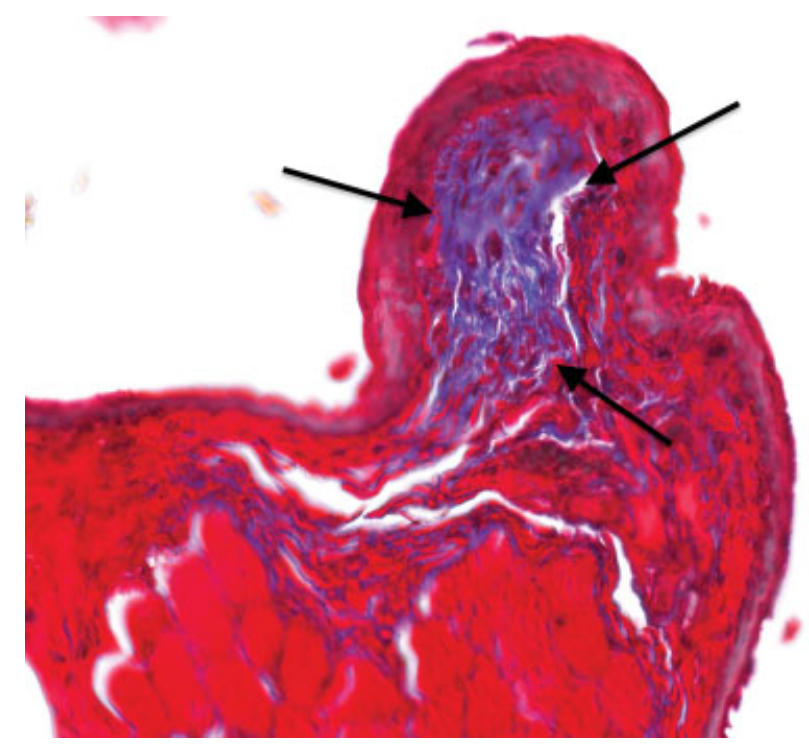

Fig. 3 Right vocal fold of a rabbit from group A with grafted fascia within the lamina propria and injected fibroblasts growth factor. Mild fibrosis and irregular deposition of collagen fibers in the subepithelial space (arrow) (Trichrome Massonx20).

fold. Reijonen et $\mathrm{al}^{19}$ injected minced fascia into the vocal muscle and observed that the graft remained identifiable histologically after 1 year of follow-up. Tsunoda et $\mathrm{al}^{20,21}$ used intact fascia graft within the Reinke's space for correction of glottal incompetence in human model, mainly arising from sulcus vocalis, with promising results.

The correction of extracellular matrix components distribution is essential to restore the structure of the vocal folds' lamina propria. These components are primarily synthesized by fibroblasts, and fascia is rich in this type of cells. Hirano et $\mathrm{al}^{22}$ demonstrated that the growth factor is a strong inducer of fibroblasts proliferation and that it stimu-

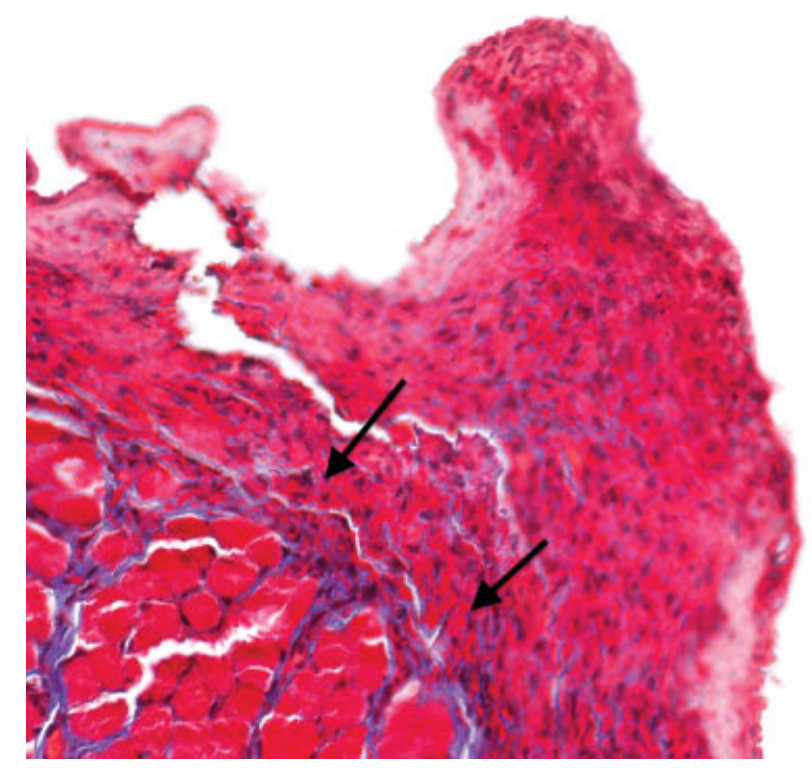

Fig. 4 Right vocal fold of a rabbit from group $B$ with grafted fascia within the lamina propria and injected fibroblasts growth factor. Mild fibrosis and irregular deposition of collagen fibers in the subepithelial space (arrow) (Trichrome Massonx20). lates the production of hyaluronic acid from fibroblasts culture. Therefore, we must assume that, when the fibroblasts are stimulated by the addition of growth factors, there is an induction of hyaluronic acid production, key for tissue repair. This study, to the best of our knowledge, is the first to histologically evaluate the use of superficial cervical fascia, associated with FGFs, when grafted into the Reinke's space of the vocal folds of a rabbit model.

The presence of vocal fold scars due to injury or inflammation can alter the ultrastructure of the lamina propria, disrupt the normal mucosal wave, and may result in glottic incompetence and dysphonia, affecting the quality of life. ${ }^{23,24}$ Several substances have been used to prevent this problem and improve glottal closure, from synthetic to biological materials: Teflon, Gelfoam (Pfizer Inc., New York, NY, USA), intralesional steroids, bovine collagen, homologous collagen, muscular fascia, and fat. ${ }^{23-25}$ Our study is the first to evaluate the fascia fragment integration into the lamina propria with the addition of FGFs.

First, the presence of a lymphohistiocytic pattern was observed in both groups (after 1 and 12 months of procedure) of grafted vocal folds (left and right). It is also important to note that the absence of macrophages among all the samples may be related to an absent activating factor, triggering macrophage activity. Robins et al $^{26}$ demonstrated that the presence of macrophage is related to foreign body chronic inflammatory reaction. However, Reijonen et $\mathrm{al}^{19}$ identified that no intense inflammatory reaction was fostered until up to 12 months after muscular fascia injection in paralyzed canine vocal folds. The isolated presence of the fascia did not trigger inflammatory reaction, as demonstrated by Duke et $\mathrm{al}^{27}$ who evaluated vocal folds that have undergone crushed fascia lata injection.

Secondly, in vitro studies showed that the FGFs led to an increased production of type I collagen and hyaluronic acid. ${ }^{5,28}$ Yet, Scapini et $\mathrm{al}^{29}$ demonstrated that their therapeutic interventions in an animal model of rabbits statistically increased the density of collagen around the graft. However, Akdogan et $\mathrm{al}^{23}$ found significant difference in a group of vocal folds from rabbits treated with vitamin A compared with an untreated group (lesser deposition of collagen and fibroblasts in the first group); and Krishna et $\mathrm{al}^{25}$ studying in vitro and in vivo vocal fold scar model of rabbits with hepatocyte growth factor exposure, found a decreased collagen secretion by fibroblasts. Our study demonstrated that the group of vocal folds injected with FGFs seemed to trigger an increased inflammatory reaction within 30 days after the procedure, compared with the group of vocal folds with no FGFs injection. Furthermore, the density of collagen observed within the vocal folds is bigger in rabbits from group $A$ than in animals from group $B$, in both sides. Our observations are similar to others found in the literature, since healing and scarring processes take more than 1 month, and mature collagen was also present during those studies. ${ }^{30,31}$

Interestingly, the grafted fascia injected with FGFs seemed to be associated to an increased rate of neovascularization, which may indicate a positive effect of grafted tissue integration on the receiver site. No preliminary work evaluated the neovascularization process in grafted vocal folds. 
Our findings also suggest that FGFs may have triggered an acute inflammatory reaction. Because there was a statistical difference between both groups and both sides of the vocal folds, we may assume that the presence of grafted fascia was not the only factor to trigger the inflammatory reaction. Only one study had evaluated the presence of inflammatory reaction after grafting fascia into vocal folds, ${ }^{27}$ with similar findings.

This is the first study to evaluate the cervical superficial fascia fragment integration into the vocal folds of rabbits with the addition of FGFs. All specimens in the longer observation group showed frequent signs of fibrosis that may have been triggered by FGFs. This may suggest that fascia grafting, used isolatedly, may become a good option for surgical procedures because it did not induce an acute or chronic inflammatory reaction or trigger fibrosis.

\section{Conclusion}

Fibroblast growth factors may have induced fibrosis and neovascularization when injected in the vocal fold of rabbits. Fibroblast growth factors alone do not represent a good therapeutic option in phonosurgery, since we observed higher levels of fibrosis in the vocal fold lamina propria. Laryngologists should be aware of this when performing phonosurgery.

\section{References}

1 Kishimoto Y, Hirano S, Suehiro A, et al. Effect of exogenous hepatocyte growth factor on vocal fold fibroblasts. Ann Otol Rhinol Laryngol 2009;118(08):606-611

2 Hirano M, Yoshida T, Tanaka S, Hibi S. Sulcus vocalis: functional aspects. Ann Otol Rhinol Laryngol 1990;99(9 Pt 1):679-683

3 Lindestad PA, Hertegård S. Spindle-shaped glottal insufficiency with and without sulcus vocalis: a retrospective study. Ann Otol Rhinol Laryngol 1994;103(07):547-553

4 Sato K, Hirano M, Nakashima T. Fine structure of the human newborn and infant vocal fold mucosae. Ann Otol Rhinol Laryngol 2001;110(5 Pt 1):417-424

5 Hirano S, Bless DM, Heisey D, Ford CN. Effect of growth factors on hyaluronan production by canine vocal fold fibroblasts. Ann Otol Rhinol Laryngol 2003;112(07):617-624

6 Hirano S, Bless DM, Rousseau B, et al. Fibronectin and adhesion molecules on canine scared vocal folds. Laryngoscope 2003; 113:969-972

7 Ohno T, French LC, Hirano S, Ossoff RH, Rousseau B. Effect of hepatocyte growth factor on gene expression of extracellular matrix during wound healing of the injured rat vocal fold. Ann Otol Rhinol Laryngol 2008;117(09):696-702

8 Hirano S, Bless DM, Massey RJ, Hartig GK, Ford CN. Morphological and functional changes of human vocal fold fibroblasts with hepatocyte growth factor. Ann Otol Rhinol Laryngol 2003;112 (12):1026-1033

9 Kanemaru S, Nakamura T, Omori K, et al. Regeneration of the vocal fold using autologous mesenchymal stem cells. Ann Otol Rhinol Laryngol 2003;112(11):915-920
10 Luo Y, Kobler JB, Zeitels SM, Langer R. Effects of growth factors on extracellular matrix production by vocal fold fibroblasts in 3-dimensional culture. Tissue Eng 2006;12(12):3365-3374

11 Thibeault SL, Rousseau B, Welham NV, Hirano S, Bless DM. Hyaluronan levels in acute vocal fold scar. Laryngoscope 2004;114(04): 760-764

12 Ornitz DM, Itoh N. The fibroblast growth factor signalling pathway. Wiley Interdiscip Rev Dev Biol 2015;4(03):215-266

13 Chan RW, Titze IR. Viscosities of implantable biomaterials in vocal fold augmentation surgery. Laryngoscope 1998;108(05):725-731

14 Chan RW, Titze IR. Hyaluronic acid (with fibronectin) as a bioimplant for the vocal fold mucosa. Laryngoscope 1999;109(7 Pt 1):1142-1149

15 Hallén L, Testad P, Sederholm E, Dahlqvist A, Laurent C. DiHA (dextranomers in hyaluronan) injections for treatment of insufficient closure of the vocal folds: early clinical experiences. Laryngoscope 2001;111(06):1063-1067

16 Rosen CA. Vocal fold scar: evaluation and treatment. Otolaryngol Clin North Am 2000;33(05):1081-1086

17 Woo P, Rahbar R, Wang Z. Fat implantation into Reinke's space: a histologic and stroboscopic study in the canine. Ann Otol Rhinol Laryngol 1999;108(08):738-744

18 Rihkanen $\mathrm{H}$. Vocal fold augmentation by injection of autologous fascia. Laryngoscope 1998;108(1 Pt 1):51-54

19 Reijonen P, Leivo I, Nevalainen T, Rihkanen H. Histology of injected autologous fascia in the paralyzed canine vocal fold. Laryngoscope $2001 ; 111(06): 1068-1074$

20 Tsunoda K, Takanosawa M, Niimi S. Autologous transplantation of fascia into the vocal fold: a new phonosurgical technique for glottal incompetence. Laryngoscope 1999;109(03):504-508

21 Tsunoda K, Niimi S. Autologous transplantation of fascia into the vocal fold. Laryngoscope 2000;110(04):680-682

22 Hirano S, Kishimoto Y, Suehiro A, Kanemaru S, Ito J. Regeneration of aged vocal fold: first human case treated with fibroblast growth factor. Laryngoscope 2008;118(12):2254-2259

23 Akdogan O, Selcuk A, Ozcan I, et al. Activation of vocal fold healing with topical vitamin A in rabbits. Acta Otolaryngol 2009;129(02): 220-224

24 de Giacomo Carneiro C, Sennes LU, Saldiva PHN, Tsuji DH, Ximenes Filho JA. Assessment of collagen deposits after implant of fascia lata and fat in the vocal folds of rabbits: histomorphometric study. Rev Bras Otorrinolaringol (Engl Ed) 2005;71(06):798-802

25 Krishna P, Rosen CA, Branski RC, Wells A, Hebda PA. Primed fibroblasts and exogenous decorin: potential treatments for subacute vocal fold scar. Otolaryngol Head Neck Surg 2006;135(06): 937-945

26 Robins SL, Cotran RS, Kumar VM. Acute and chronic inflammation. In: Robins SL, Cotran RS, Kumar VM, eds. Structural and functional pathology. Rio de Janeiro, RJ: Ed. Guanabara; 1886:680-696

27 Duke SG, Salmon J, Blalock PD, Postma GN, Koufman JA. Fascia augmentation of the vocal fold: graft yield in the canine and preliminary clinical experience. Laryngoscope 2001;111(05):759-764

28 Branski RC, Barbieri SS, Weksler BB, et al. Effects of transforming growth factor-beta1 on human vocal fold fibroblasts. Ann Otol Rhinol Laryngol 2009;118(03):218-226

29 Scapini F, da Silva LF, Tsuji DH, Dolhnikoff M, Sennes LU. Effect of fibrin glue on collagen deposition after autologous fascia grafting in rabbit vocal folds. Ann Otol Rhinol Laryngol 2011;120(10):663-668

30 Rousseau B, Hirano S, Chan RW, et al. Characterization of chronic vocal fold scarring in a rabbit model. J Voice 2004;18(01):116-124

31 Thibeault SL, Gray SD, Bless DM, Chan RW, Ford CN. Histologic and rheologic characterization of vocal fold scarring. J Voice 2002;16 (01):96-104 